\title{
Construcción de la dimensión cognitiva de alfabetización científica en el estudiantado, a través de las Olimpiadas Costarricenses de Ciencias Biológicas
}

\section{Construction of the Cognitive Dimension of the Scientific Literacy in the Students through the Costa Rican Biological Sciences Olympics}

\author{
Shirley Camacho Vargas ${ }^{1}$ \\ Escuela de Ciencias Biológicas \\ Universidad Nacional \\ Heredia, Costa Rica \\ shircv.26@gmail.com \\ José Pereira Chaves ${ }^{2}$ \\ Escuela de Ciencias Biológicas \\ Universidad Nacional \\ Heredia, Costa Rica \\ jpereira@una.ac.cr
}

Recibido 11 de noviembre de 2011 • Corregido 21 de mayo de 2012 • Aceptado 28 de junio de 2012

Resumen. En esta investigación se reconocen las contribuciones cognitivas al estudiantado participante de la III Olimpiada Costarricense de Ciencias Biológicas que determinan avances y fortalecimiento en la construcción de su dimensión conceptual de alfabetización científica. Este trabajo se basó, predominantemente, en técnicas del enfoque cualitativo (diseño etnográfico: estudio de caso); sin embargo, algunos datos se interpretan mediante metodologías de corte cuantitativo (diseño descriptivo con un matiz explicativo y exploratorio) para el análisis de una muestra de 54 estudiantes de secundaria, finalistas en la categoría A de dichas competencias, con el empleo de instrumentos tales como un estudio documental y una encuesta, en julio del 2009. La información generada se analizó utilizando elementos de estadística inferencial y descriptiva, además de figuras e histogramas. Se comprobó que se genera un mejor manejo cognitivo en las temáticas evaluadas, un aumento en el rendimiento estudiantil conforme la aplicación de las pruebas, un compromiso por la actualización académica apoyado por el desarrollo de diversas labores de preparación previa, aportes curriculares inéditos sobre esta muestra, una anuencia por optimizar sus conocimientos referentes a la Biología, lo cual permitiría aplicar nociones científicas al diversificar y renovar los conocimientos, según lo planteado en los principios de la alfabetización científica.

Palabras claves. Alfabetización científica, dimensión conceptual, dimensión cognitiva, Olimpiadas Costarricenses de Ciencias Biológicas.

\footnotetext{
Licenciatura y Bachillerato en Enseñanza de las Ciencias Naturales y Bachillerato en Biología con énfasis en Biología Tropical, de la Universidad Nacional, Co-coordinadora de las Olimpiadas Costarricense de Ciencias Biológicas e internacionales, académica investigadora en el área de Enseñanza de las Ciencias Naturales de la Escuela de Ciencias Biológicas de la Universidad Nacional, Heredia, Costa Rica. Apartado 86-3000.

2 Candidato a Doctor en Educación, de la Universidad Estatal a Distancia, Costa Rica. Maestría en Ciencias Marinas, de la Universidad Nacional (UNA), Licenciatura en Ciencias de la Educación con énfasis en Didáctica de las Ciencias, Bachillerato en Enseñanza de las Ciencias Naturales Universidad Nacional de Costa Rica, Coordinador de las Olimpiadas Costarricense de Ciencias Biológicas e internacionales y de la Comisión de trabajos finales de graduación de la Maestría en Ciencias Marinas de la Escuela de Ciencias Biológicas, profesor del área de Enseñanza de las Ciencias e investigador el área de Biología Marina, Universidad Nacional, Heredia, Costa Rica. Apartado 86-3000.
} 
URL: http://www.una.ac.cr/educare

\begin{abstract}
This research recognizes the cognitive contributions to the students participating in the Third Costa Rican Biological Sciences Olympics that will define the advancement and strengthening in the construction of its conceptual dimension in the scientific literacy. This paper is based, mainly, on qualitative approach techniques (ethnographic design: case study); however, some data are interpreted through quantitative methodologies (descriptive design with an explanatory and exploratory touch) for the analysis of a sample of 54 high school students, finalists in the category A of the Olympics, through the use of tools such as a documentary study and a survey, in July 2009. The information generated was analyzed using elements of inferential and descriptive statistics, figures and histograms. It was proved that there is a better cognitive management in the topics assessed, an increase in the students' academic performance as the tests are applied, a commitment for the academic update supported by the development of several tasks for previous preparation, curriculum contributions unprecedented based on our sample, a consent to optimize student's knowledge about Biology, which will allow the application of scientific notions to diversify and renew the knowledge, according to what is established in the principles of scientific literacy.
\end{abstract}

Keywords. Scientific literacy, conceptual dimension, cognitive dimension, Costa Rican Biological Sciences Olympics.

\title{
Estado de la cuestión
}

Los comienzos de la alfabetización científica (AC en adelante) se remontan a la década de 1980, cuando países desarrollados como Estados Unidos e Inglaterra realizaron esfuerzos por determinar y analizar el nivel de esta en sus poblaciones. Los resultados fueron tan sorprendentes que lideraron estrategias para aumentar, en sus sociedades, el conocimiento científico básico, el involucramiento con su accionar y la sensibilización ante las Ciencias (Johnson, 1989; Miller, 1983).

Sin embargo, los estudios sobre cómo se emplea la Ciencia en situaciones reales o cotidianas aún son escasos y deberían responder a la necesidad de alfabetizar científicamente para lograr que la ciudadanía ejerza, con un criterio pertinente, sus deberes y derechos en decisiones relacionadas a ella (Cajas, 2001). Este autor también manifiesta que la AC es una novedosa ideología en el rediseño del discurso científico escolar. Esta, al aumentar la coherencia y relevancia de lo que se enseña en la cotidianidad de cada individuo, pretende desarrollar Ciencia para todos y todas, no solo para quienes van a ser científicos profesionales.

Consecuentemente, estatendencia, enesencia, promueve una cultura donde secomprendan las verdades científicas dentro la formación ciudadana, brindándole carácter humanitario-social y notable a la Ciencia (Reyes y Molina, 2005); revalorando el desarrollo humano sostenible y el papel de las instituciones científicas en él (Acevedo, Vázquez y Manassero, 2003).

La AC ha conseguido destacarse socialmente en muchos ámbitos y esto se evidencia en el gran número de investigaciones, publicaciones, congresos y encuentros que han surgido con el lema "Ciencia para todos" (Marco-Stiefel, 2000) y en las múltiples reformas educativas que se llevan a cabo en cuantiosos países y que la contemplan como una finalidad. 
Así, los investigadores resaltan que una educación, regida por una cultura científica básica y por la AC, debería contener conocimientos, aplicaciones científicas, habilidades, tácticas, resolución de problemas, interacción con tecnología, cuestiones socioeconómico-políticas además de ético-morales en la Ciencia, su historia y desarrollo, estudio de la naturaleza y la práctica científica (Acevedo y Acevedo, 2007; Bybee, 1997; Vázquez, Manassero).

De este modo, en el ámbito latinoamericano surgieron designios encaminados al logro de dichos objetivos. Específicamente, en Argentina se instauró el Proyecto de Alfabetización Científica (2007), a cargo de la Dirección Nacional de Gestión Curricular y Formación Docente del Ministerio de Educación, Ciencia y Tecnología de la República Argentina.

De la misma forma, otra iniciativa destacable es la denominada: "¿Cómo promover el interés por la cultura científica? Una propuesta fundamentada para la educación científica de jóvenes de 15 a 18 años" de la Oficina Regional de Educación para América Latina y el Caribe, Organización de las Naciones Unidas para la Educación, la Ciencia y la Cultura (UNESCO) presentada durante el 2005 con pautas contextualizadas para el impulso de la AC.

A pesar de ello, en Costa Rica son prácticamente nulas las iniciativas evaluadoras del papel de la extensión universitaria propiamente en la AC a nivel preuniversitario, o que sustenten las bases referentes a diligencias ajustadas a este marco. Por ello, resulta válido, desde la capacidad potencial de estas cuestiones, utilizar los resultados y conclusiones de este trabajo, para transformar contenidos curriculares y mejorar la comprensión pública de la Ciencia, desde la perspectiva de participación en las Olimpiadas Costarricenses de Ciencias Biológicas (OLICOCIBI, en adelante).

Las OLICOCIBI, desde su visión, se ha concebido como un programa que promueve el estudio activo, participativo y significativo de las Ciencias Biológicas a nivel preuniversitario. Además, como una actividad inclusiva, que considera todas las instituciones públicas y privadas, en sus diferentes modalidades y abarca todas las sedes regionales. Igualmente, la misión de trabajo que guía sus acciones pretende impulsar el interés por el estudio de las Ciencias Biológicas, con el fin de contribuir al desarrollo científico y tecnológico del país.

De tal modo, en consideración con su visión y misión así como por sus objetivos, el proyecto OLICOCIBI forja la educación biológica, desde una concepción que coincide con la exigencia de la AC, consensuada en la Conferencia Mundial sobre la Ciencia para el Siglo XXI, donde se declara que es un imperativo estratégico lograr que el estudiantado resuelva problemas concretos y atienda las necesidades sociales mediante sus competencias y conocimientos científicos; además, que es necesario fomentar y difundir la AC en todos los ámbitos sociales para mejorar la participación ciudadana en la adopción de disposiciones relativas a la aplicación de la Ciencia (Declaración de Budapest, 1999).

Precisamente, las OLICOCIBI surgidas en el año 2007, por el interés del Ministerio de Ciencia y Tecnología (MICIT en adelante) y de sectores académicos de la Universidad Nacional (UNA en adelante), pretenden que el alumnado y profesores tutores adquieran -o al menos 
URL: http://www.una.ac.cr/educare

fortalezcan- cierta comprensión y apreciación global por la Ciencia desde la posición de la AC, gracias a la apertura de un espacio de competencia cognitiva o concurso académico que evalúa el desempeño del estudiantado frente a la resolución de pruebas teóricas y prácticas referentes a las Ciencias Biológicas. Esta faculta en su proceso, para el estudiante, la posibilidad de identificar, interpretar, argumentar y resolver problemas del contexto relacionados con las Ciencias Biológicas; además, procura fomentar comportamientos formados por habilidades cognitivas, actividades de valores, destrezas y diversas informaciones que hacen posible llevar a cabo, de manera eficaz, el aprendizaje de la Biología y sus aplicaciones.

Por lo tanto, se intenta reconocer las contribuciones cognitivas, en el estudiantado, tras su participación en estas competencias académicas. El propósito es determinar cuál es el fortalecimiento en su dimensión conceptual o cognitiva, según la AC. De esta manera, poder promover la instauración y consolidación de un modelo de desarrollo que contribuya al mejoramiento de la comunidad científica en el campo de las Ciencias Biológicas del país.

Finalmente, para la educación secundaria costarricense, las iniciativas que evalúen la AC ligada a la participación en espacios extracurriculares como las OLICOCIBI, simbolizarían la generación de estrategias que fomenten la motivación para actualizar y ampliar conocimientos en las Ciencias Biológicas, la adquisición y diversificación de habilidades y la construcción de actividades que permitan transversalizar contenidos temáticos con una realidad fuera de las aulas.

\section{Marco teórico}

De acuerdo con lo establecido en la Conferencia Mundial sobre la Ciencia para el Siglo XXI, la AC en la educación se evidencia cuando el estudiantado es capaz de resolver problemas concretos atendiendo las necesidades sociales, utilizando sus habilidades y conocimientos científico-tecnológicos, a fin de mejorar la participación ciudadana en la adopción de decisiones relativas a la Ciencia. Se estipula como las capacidades potencializadas en estudiantes con un fin claro de participación social.

Furió, Vilches, Guisasola y Romo (2001) señalaron que su nacimiento corresponde a un hito reciente en las reformas curriculares de la educación científica y se centran en la inclusión de componentes en el currículo, para orientar su enseñanza hacia aspectos sociales y personales de del propio estudiante; estas relaciones enfatizan la necesidad de una Ciencia para todos, esencial en la formación básica ciudadana, la que no puede alejarse ni añadírsele como una exclusividad a los currículos escolares. Cabe destacar que estos movimientos aclaran que la AC es algo a lo que se aspira y que se desarrolla durante la vida, pero que en esencia se favorece en etapas de formación escolar (Fensham, 1985).

Laugksch (2000) califica a la AC como la capacidad de utilizar el conocimiento científico para entender y participar en la toma de decisiones sobre el mundo natural y los cambios realizados en él a través de la actividad humana. 
Para esta investigación se consideró, además de las nociones mencionadas, un concepto integrador que involucra los principios de Shamos (1995), Kemp (2002) y Acevedo et al. (2003), al reconocer que la AC es una ideología en la enseñanza científica, que transforma el currículo educativo y formativo social, al procurar la comprensión, el uso de un rango amplio de conceptos o vocabulario científico en la cotidianidad y cultura propia, con propósitos cívicos, democráticos además de sociales, por parte de todas las personas, independientemente de la profesión que ejerzan o de sus gustos académicos.

Las perspectivas de accionar que la AC adquiere (Reyes y Molina, 2005) armonizan con las orientaciones de la labor en las OLICOCIBI. Corresponden, en un primer sentido, al político, porque las formas de leer el mundo e interpretar las situaciones están inmersas en relaciones de poder. En segundo, al ético, porque los sujetos leen el mundo según circunstancias de clase, género, raza y actitud política, así como de contextos y relaciones sociales. Por último, cultural porque está inmerso en redes de significancia construidas por el propio sujeto en su afán de interpretar el mundo. Aguilar (1999) expresa que indiscutiblemente dichos sentidos añaden implicaciones pedagógicas, tanto para la formación ciudadana como para la de maestros.

Cajas (2001) y Collins (1997) expusieron que la AC se caracteriza por una serie de elementos que intentan promover un discurso científico escolar más coherente y relevante. Acevedo et al. (2003) y Kemp (2002) añaden elementos o rasgos deseables sobre la AC y los agrupan en tres dimensiones: conceptual (comprensión de conocimientos), procedimental (procedimientos, procesos, habilidades y capacidades), afectiva (emociones, actitudes, valores y disposición).

El parámetro fundamental de estudio en esta investigación es la dimensión conceptual o cognitiva de AC, en la que sobresalen la apropiación, el uso y la comprensión de los conceptos científicos y cómo estos se relacionan con la sociedad. Además, otros rasgos que definen esta dimensión son la disposición en ampliar el manejo cognitivo y la mejoría sustancial en el conocimiento referente a tópicos científicos (Kemp, 2002).

Del mismo modo, este autor estableció tres tipos de AC: personal, práctica y formal, que incorporan diferentes particularidades de las dimensiones citadas.

En la AC personal destacan elementos de la dimensión conceptual y razones de escala individual, que subrayan rasgos de la dimensión afectiva. Desde esta perspectiva, radica en comprender un amplio rango de conceptos y usar un extenso vocabulario científico cotidianamente, apreciar la historia de la Ciencia, comprender su divulgación, interesarse por ella y estar motivado para seguir aprendiéndola después de la escolarización formal.

En la AC práctica se hace hincapié en rasgos de la dimensión procedimental y justificaciones de la escala práctica; en segundo plano la dimensión afectiva. Desde este punto de vista, consiste en saber usar la Ciencia en la vida cotidiana, con propósitos cívicos y sociales, obtener información sobre ella, comprender su divulgación y los mensajes de medios de comunicación, entender las relaciones Ciencia-Sociedad, conocer conceptos básicos y apreciarla siendo consciente de sus limitantes. 
URL: http://www.una.ac.cr/educare

Por último, la AC formal incluye rasgos de todas las dimensiones, a escala individual y práctica; lo que implica conocer conceptos científicos, tener una amplia comprensión de sus principios, saber sobre su naturaleza y las relaciones Ciencia-Sociedad, obtener información científica, utilizarla y ser capaz de comunicarla, ser competente al usarla en la vida cotidiana y participar democráticamente en la sociedad civil en la toma de decisiones sobre asuntos relacionados con la Ciencia y tecnología, apreciarla, interesarse por ella y estar al día de sus novedades.

Adicionalmente, Reid y Hodson (1993) propusieron elementos que la educación dirigida hacia una cultura científica debería contener. Sobre algunos de ellos se comprobó la promoción de la dimensión conceptual de la AC en esta investigación, los cuales son:

- Historia y desarrollo de la Ciencia.

- Conocimientos de la Ciencia (hechos, conceptos y teorías).

- Cuestiones socio-económico-políticas y ético-morales en la Ciencia.

- Aplicaciones del conocimiento científico (uso en situaciones reales y simuladas).

- Resolución de problemas (aplicación de habilidades, tácticas y conocimientos a investigaciones reales).

- Habilidades y tácticas de la Ciencia (familiarización con los procedimientos, uso de aparatos e instrumentos).

- Estudio de la naturaleza y la práctica científica (consideraciones filosóficas y sociológicas de métodos científicos).

- Interacción con la tecnología (resolución de problemas prácticos, enfatización científica, estética, económica, social y aspectos utilitarios de las posibles soluciones).

En este trabajo se analizaron los aportes a la dimensión conceptual lograda mediante los tipos de AC personal, práctica y formal, desde sus diferentes concepciones en la participación del estudiantado.

Como señalan Hicks y Holden (1995), para que el estudiantado asuma una ciudadanía responsable en su $A C$, es preciso proporcionarle herramientas y espacios para el análisis de problemáticas globales y la consideración de las posibles soluciones durante su formación. Con las OLICOCIBI se pretende abrir un espacio para lograr la comprensión significativa de los conceptos y plantear el aprendizaje de las Ciencias como algo próximo a la investigación científica, que sirva de puente para la AC.

De acuerdo con los orígenes, propuesta y su normativa, estipulados por su Comisión Organizadora (Olimpiadas Costarricenses de Ciencias Biológicas [OLICOCIBI], 2011), la OLICOCIBI constituye una competencia intelectual dirigida a jóvenes estudiantes del III Ciclo de la Educación General Básica y del Ciclo Diversificado, cuyo propósito primordial es estimular el estudio de las Ciencias Biológicas y descubrir jóvenes talentosos en esta disciplina. Asimismo favorece el despertar de talentos científicos como futuro potencial de desarrollo en el país. 
En cuanto al proceso de participación y selección (normativa, capítulo III), los participantes y las participantes eligen, al momento de la inscripción, la categoría en la cual desean competir, según los requerimientos de cada una de ellas $y$, evidentemente, por su posible afinidad o interés con las Ciencias Biológicas.

Para presentarse en la categoría A, deben ser estudiantes regulares de Educación Secundaria en cualquiera de sus modalidades, idealmente no deben haber cumplido 18 años al $1^{\text {ero }}$ de octubre del año de la competencia.

Para la categoría $B$, deben ser estudiantes regulares de sétimo, octavo, noveno o décimo años. En el caso de instituciones de la modalidad técnica, podrán participar los de undécimo año. La participante o el participante no debe haber ganado medalla de oro en esta categoría en años anteriores al de la convocatoria ni haber participado tres veces en este nivel de competencia.

Cada institución educativa inscribe un máximo de 5 estudiantes en cada categoría.

Quienes participan realizan una prueba teórica eliminatoria individual regional. Aproximadamente un $20 \%$ de la población participante en cada categoría y, según el rendimiento, avanza a la segunda etapa.

La fase final de la categoría A consta de dos pruebas: una teórica escrita y otra experimental; la primera, de evaluación individual, tiene una duración aproximada de tres horas (para un valor de $70 \%$ del total) y la segunda, de evaluación grupal, se desarrolla en una de las Estaciones Experimentales de la Escuela de Ciencias Biológicas de la Universidad Nacional, con una duración 12 horas - dos días- (30\% del valor total). La parte experimental se evalúa según la organización en el trabajo de campo, el trabajo escrito tipo artículo científico presentado sobre la labor práctica y la exposición de lo ejecutado.

La participación en espacios competitivos a nivel educativo como la OLICOCIBI responde a la necesidad de socializar fuera de las aulas y vincular el conocimiento, las destrezas y habilidades adquiridas, con la realidad contextualizada del estudiantado (Informe Técnico presentado al CONICIT, 2008); lo cual coincide con lo expuesto como fines propios de la AC.

\section{Metodología}

Este trabajo se guió con modelos que constituyen una concepción intermedia entre los principios teóricos que fundamentan la investigación y sus procedimientos (Alvira, 1983), emplea nociones del paradigma positivista e interpretativo (Barrantes, 1999; Bourdieu, Chamboredon y Passeron, 2002; García, 1987).

Asimismo, se enmarcó en un enfoque mixto, desde el cual se utilizaron técnicas de recolección y análisis de datos basadas en aspectos cuantitativos y cualitativos. 
Mediante elementos del enfoque cuantitativo se recurrió a la recolección de datos para probar hipótesis, basados en mediciones numéricas, análisis estadísticos, establecimiento de patrones de comportamiento y prueba de teorías (Hernández, Fernández y Baptista, 2003).

Según el enfoque cuantitativo, este trabajo se considera descriptivo de los fenómenos que fomentan la dimensión cognitiva de AC en el proceso de participación en las OLICOCIBI.

A pesar de ello, no debe olvidarse que se pretende situar la AC como un componente derivado de la participación en estas competencias, por lo que se inicia como el primer estudio con dichos alcances, con lo cual se obtiene un matiz exploratorio.

También se hizo empleo primordial del enfoque cualitativo, para la interpretación de datos obtenidos y el establecimiento de propuestas concluyentes o teorías al finalizar la misma, con la contrastación empírica y en la medición objetiva de fenómenos (Martínez, 2001).

Desde este enfoque, esta investigación se fomenta con un diseño etnográfico específicamente considerado como un estudio de caso, referente a la construcción de la dimensión conceptual de AC en los estudiantes participantes finalistas.

Los sujetos fuente de información en este trabajo corresponden a los estudiantes y las estudiantes participantes en la III OLICOBICl${ }^{3}$, de categoría A, provenientes de distintas regiones y modalidades educativas del país. Es una muestra heterogénea, representativa, estadísticamente significativa y elegida según el criterio de calificaciones superiores o iguales a las necesarias para participar en la etapa final, además, debido a la facilidad de acceso durante la fase final. Contabilizan 54 estudiantes (30 mujeres y 24 hombres), que comprenden un conjunto de jóvenes de entre 16 a 19 años, estudiantes de XI y XII nivel de secundaria del Sistema Educativo Costarricense.

Se revisaron fuentes primarias como libros y revistas referentes a la temática que rige la misma. También, información recolectada de los instrumentos diseñados para la descripción directa e indirecta de la muestra. Igualmente, se consultó bibliografía de catálogos, bases de datos, buscadores y webs en internet como fuentes secundarias (Carrizo, Irureta-Goyena y López 2000).

Debido a las particularidades de las OLICOCIBI, las fuentes institucionales las constituyen los colegios cuyos representantes lograron pasar a la etapa final y pertenecen a 3 modalidades (8 científicos, 23 académicos y 1 técnico) de diferentes subvenciones económicas (8 privados, 2 semiprivados y 22 públicos).

Para corroborar los aportes de la dimensión conceptual en el alumnado, se realizó un análisis documental, específicamente comparando los resultados y el rendimiento en la prueba teórica eliminatoria frente a la final, considerándose como avance cognitivo. Así, se estudió el rendimiento en las fases de manera objetiva, sistemática y cuantitativa, lo que confronta comportamientos en distintas categorías, en este caso, cognitivos (Alvira, 1983).

3 Proceso que inició en febrero con la divulgación, en marzo se registró la inscripción, en mayo y julio se ejecutaron las distintas pruebas y culminó en agosto con la premiación. 
Se aplicó una encuesta de 5 cuestionamientos sobre el nivel evaluativo en las OLICOCIBI versus el bachillerato y el del centro educativo, la posición respecto a la incorporación de otras temáticas diferentes a las propuestas por el MEP y la realización de actividades extracurriculares para competir y, finalmente, los aportes de nuevos conocimientos o el refuerzo de los previos a través de la participación en estas competencias académicas.

Los instrumentos se aplicaron durante julio del 2009, en la ejecución de la fase práctica de la prueba final de la III edición de las OLICOCIBI en la Estación de Ciencias Marino Costeras.

Algunos datos se exploraron con un análisis descriptivo y se sistematizaron mediante los programas estadísticos SPSS Statistics 17.0 (Stadistical Package for the Social Sciencies) y Excel de Microsoft.

Asimismo, se compararon elementos de estadística inferencial como el $t$-student y descriptiva, se emplearon figuras e histogramas, se contrarrestaron los grupos de frecuencias observadas (muestreales) en las respuestas a los instrumentos.

\section{Resultados, análisis y discusión}

Para explorar los aportes que se derivan de la participación en la OLICOCIBI a la dimensión conceptual de AC en el estudiantado, se consideró que las notas obtenidas por los competidores y las competidoras, en ambas fases, son indicativas del manejo académico de los contenidos, dado que involucran preparación didáctica previa para realizar ambos exámenes. Por ello, para comparar el rendimiento en las dos fases, se realizó un escrutinio de estadística descriptiva (tabla 1).

Tabla 1

Diferencias estadísticas entre el rendimiento académico del estudiantado finalista de categoría A $(n=54)$ entre la fase eliminatoria y final de la III OLICOCIBI, 2009

\begin{tabular}{lcc}
\hline \multicolumn{1}{c}{ Estdístico } & Eliminatoria & Final \\
\hline Tamaño de muestra $\mathrm{n}$ & 54 & 54 \\
\hline Promedio & 66,63 & 73,19 \\
\hline Mediana & 66,00 & 70,00 \\
\hline Desviación estándar & 5,50 & 7,25 \\
\hline Mínimo & 61,00 & 65,00 \\
\hline Máximo & 83,00 & 89,00 \\
\hline Error Estándar & 0,75 & 0,99 \\
\hline
\end{tabular}

Nota: Datos recopilados del rendimiento de las fases eliminatoria y final de la III OLICOCIBI por los autores de este trabajo. 
URL: http://www.una.ac.cr/educare

Asimismo, de acuerdo con los datos anteriores, en una $\mathrm{n}=54, \mathrm{gL}=53, \mathrm{p}<0,05$, mediante una prueba t-student, se comprobó que hay evidencia estadística significativa en el grupo de datos, por lo tanto, el rendimiento entre ambos exámenes fue distinto (figura 1). Igualmente, mediante la misma prueba estadística, se revelaron diferencias confiables entre la nota promedio obtenida de ambos exámenes ( $\mathrm{t}(53)=6,65, \mathrm{gL}=53, \mathrm{p}<0.05, \mathrm{a}=0.05$ ), por lo que se indica que hubo un aumento en las calificaciones obtenidas $y$, por ende, el rendimiento fue superior durante la fase final.

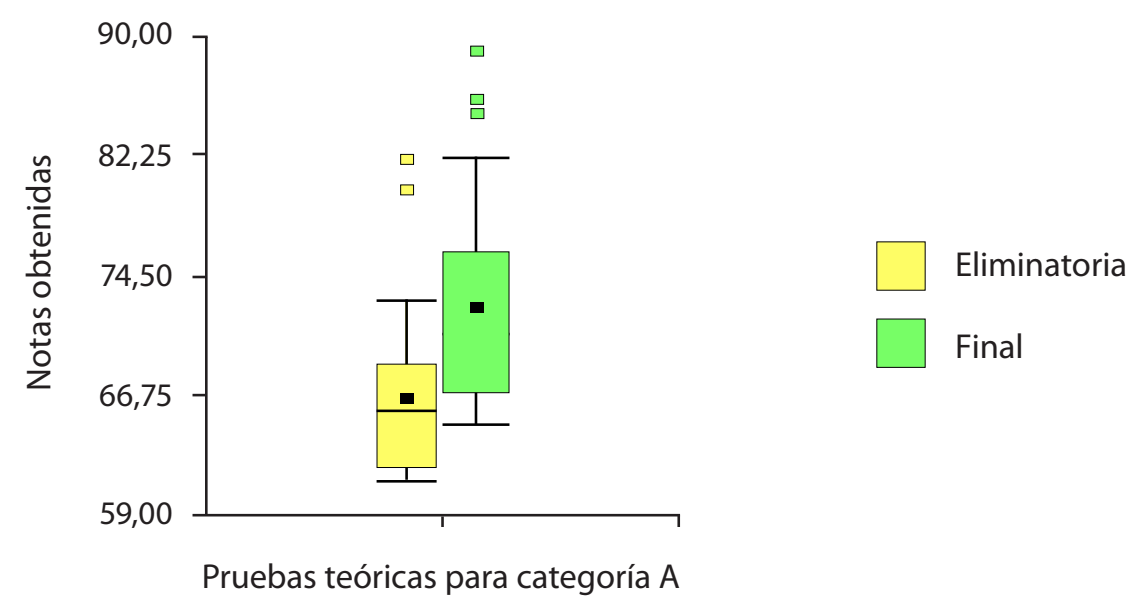

Figura 1. Rendimiento académico obtenido por el estudiantado de categoría A durante la fase eliminatoria y final de la III OLICOCIBI, 2009.

Nota: Datos recopilados del rendimiento de las fases eliminatoria y final de la III OLICOCIBI por los autores de este trabajo.

Al respecto, se resaltan varias particularidades, entre ellas:

- En ambos exámenes se repetía la evaluación de aproximadamente un 30\% de los objetivos del temario.

- El grado de complejidad en los objetivos evaluados, y según el formato de estructuración de las preguntas, era mayor en la etapa final.

- Específicamente, la fase final también presenta un incremento en el grado de dificultad, dado que en ella se evalúan más objetivos y se incorpora una sección de desarrollo en la resolución que aporta un $25 \%$ de la nota consignada a la parte escrita, según lo estipulado en el reglamento de la OLICOCIBI.

Pese a estas indicaciones, debido a que el rendimiento del estudiantado fue más alto durante la segunda etapa, se considera que hubo una consecuente mejoría en el manejo cognitivo de las temáticas evaluadas. 
Podría inferirse que este aumento también se relaciona con otras causas, tales como una mayor motivación del estudiantado por avanzar a la segunda etapa del evento, más tiempo para la preparación académica, familiarización con el tipo de evaluación, diversificación en las actividades de sustento para competir, aumento en el apoyo docente, así como el involucramiento de parte del profesorado de sus centros en el proceso final, entre otras. Estas acciones, al facilitar el diálogo alfabetizador, generarían un fomento cognoscente en el estudiantado (Giroux, 1997; Kemp, 2002) y ello se demuestra con un mejor rendimiento; lamentablemente, esta investigación no tiene el alcance de predecir o concluir sobre las estrategias que influyen de alguna forma en el rendimiento de las pruebas.

Por otro lado, se describió la percepción estudiantil de los aportes cognitivos forjados por su participación en las OLICOCIBI, en cuanto a la dificultad, generación o reconstrucción conceptual de temáticas biológicas.

Se estableció el nivel evaluativo de las OLICOCIBI con referencia a la prueba de bachillerato oficial, al considerar la preparación recibida para efectuar estas pruebas y el nivel propio de cada centro educativo (figura 2).

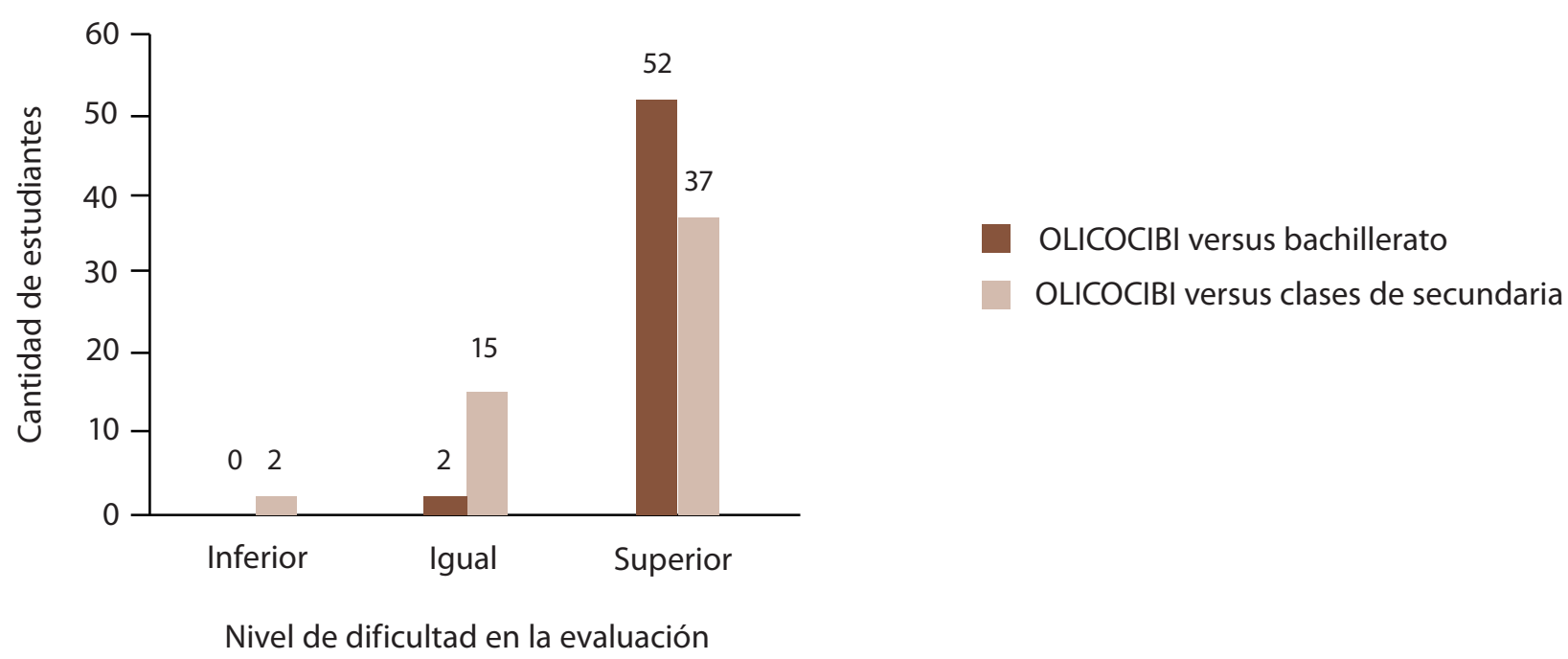

Figura 2. Número de estudiantes según el nivel de dificultad de la evaluación teórica de las OLICOCIBI versus bachillerato y clases de secundaria según el estudiantado.

Nota: Datos recopilados del instrumento "Evaluación de los aportes cognitivos de la OLICOCIBI, dirigida a estudiantes" por los autores de este trabajo, agosto, 2009.

El nivel evaluativo, tanto en comparación con lo valorado en bachillerato como en los centros educativos, se consideró mayor en las OLICOCIBI. Esto representa, inherentemente, aportes sobre contenidos conceptuales que resultan ser novedosos para los competidores, quienes al estudiarlos sobrepasan el campo cognitivo abarcado en su preparación para las pruebas de bachillerato y en sus clases habituales de secundaria. 
URL: http://www.una.ac.cr/educare

Su participación en este espacio competitivo, a pesar de que les exija un nivel de preparación académica mayor, refleja, según los principios de la $A C$, un compromiso en comprender -repasar, estudiar, analizar- aspectos teóricos de la Biología, más allá de una simple memorización, que procura fomentar el razonamiento, lo cual es determinante para la construcción de su dimensión cognitiva (Vázquez et al. 2007).

Por otro lado, dado que el Sistema Educativo Nacional se rige en el cumplimiento del currículo establecido por el MEP, se consideró la concordancia del estudiantado en cuanto a la adición de temáticas no contempladas por dicho ente y que aparecen en el temario de las OLICOCIBI. Ante esto, 49 encuestados indicaron estar de acuerdo (representando un 91\%), contrario a lo manifestado por 5 estudiantes (el 9\% restante). Las justificaciones pertinentes se clasificaron en los estratos que se indican en la figura 3.

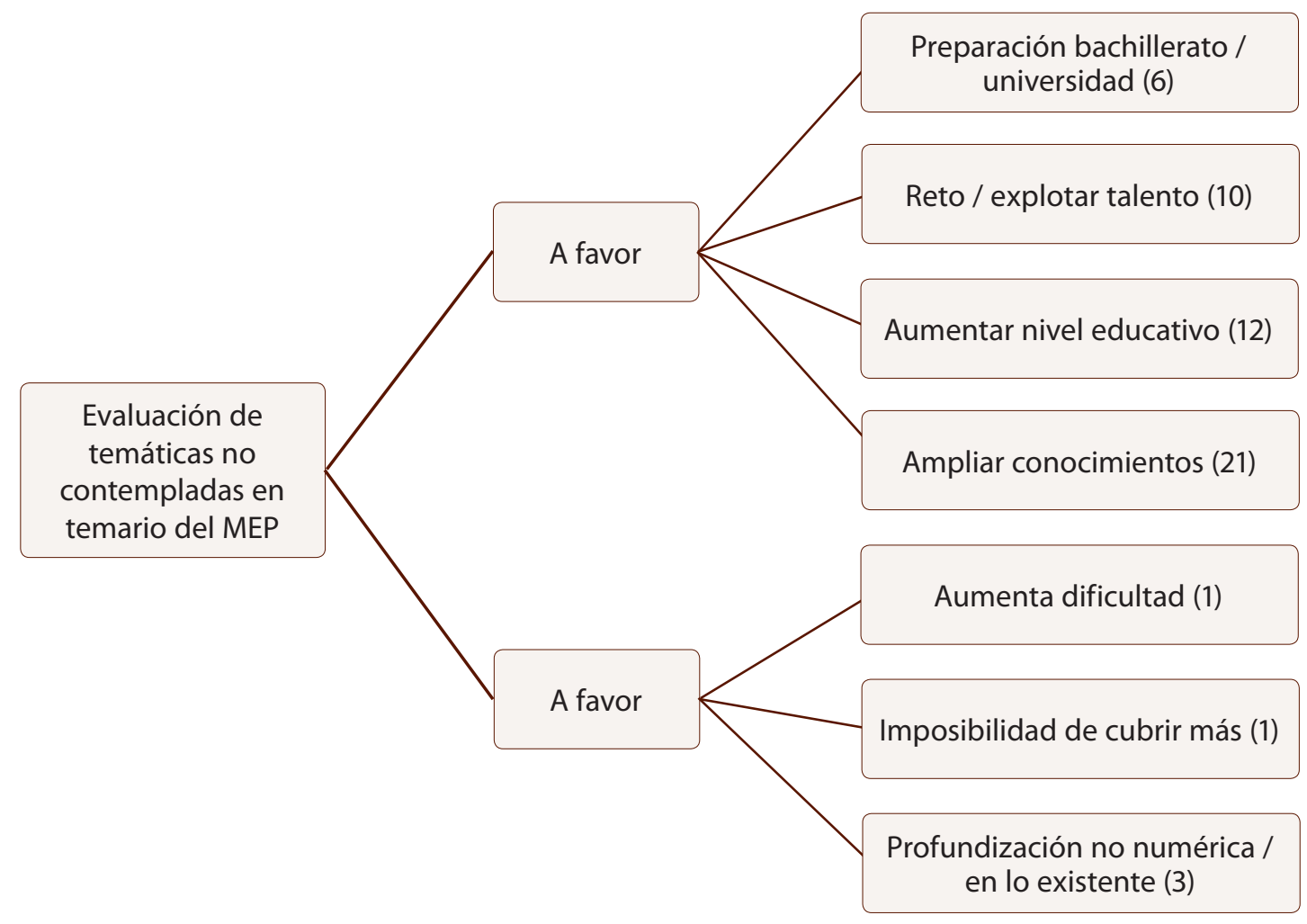

Figura 3. Razones que justifican la concordancia o no con la evaluación en las OLICOCIBI de temáticas no contempladas en el temario oficial del MEP (se indican entre paréntesis la cantidad de encuestados que expresaron cada punto de vista).

Nota: Datos recopilados del instrumento "Evaluación de los aportes cognitivos de la OLICOCIBI, dirigida a estudiantes" por los autores de este trabajo, julio, 2009. 
Cabe destacar que en los temarios de la OLICOCIBI se ha incorporado casi un 20-30\% de contenidos no abarcados por el MEP; debido a que la Comisión los contempló como elementos conceptuales de integración y complemento para el domino conceptual que los participantes deben manejar el terminar el proceso de Olimpiadas, por tanto, estos no son formal ni obligatoriamente estudiados en los centros educativos, y se convierten, por tanto, en temáticas que, muy probablemente, no se estudiarían en el nivel formativo en cuestión. Por lo tanto, su estudio se consideró como un aporte original de forma directa sobre la dimensión cognitiva del alumnado que participa en dichas competencias, el cual, al terminar el proceso, tendrá una mayor visión académica en las ciencias biológicas.

El apoyo manifestado a la evaluación de temas que no están en las directrices del MEP demuestra que, con la participación en este evento, el estudiantado aumenta su anuencia por optimizar y diversificar su manejo cognitivo de la Biología. Lo anterior, primordialmente, debido a que le permite ampliar conocimientos y la calidad educativa en que se desenvuelve, además, a que representa un reto para explotar su capacidad o talento $y$, finalmente, como complemento a las pruebas de bachillerato y de entrada a las universidades estatales.

De esta forma, con su participación en las OLICOCIBI, el estudiantado es partícipe activo del favorecimiento en el uso y la comprensión de la Ciencia para actuar en la sociedad, dado su interés por aprender más sobre ella, aún después de la escolarización formal (Kemp, 2002; Vázquez et al. 2007), lo cual constituye otro aporte a su dimensión conceptual de AC.

Otro rubro evaluado fue el cumplimiento de diversas actividades extracurriculares como apoyo para el desempeño en esta competencia académica, dado que estas aumentan y diversifican la cantidad de conocimientos en el estudiantado. Por ello, se cuestionó quiénes realizaban tales labores; solamente un 31\% de los estudiantes admitieron no cumplir con ninguna acción extracurricular frente a un $69 \%$ de los encuestados que sí las practicaban, en esta submuestra, ello se catalogó como un aporte cognitivo.

Se referencian las actividades de este 69\%; las cuales se clasificaron en tres criterios: Estudio independiente (con ayuda de un mentor o sin esta), giras y centros de estudio (acompañados por mentores externos, docentes o compañeros) (figura 4). 
URL: http://www.una.ac.cr/educare

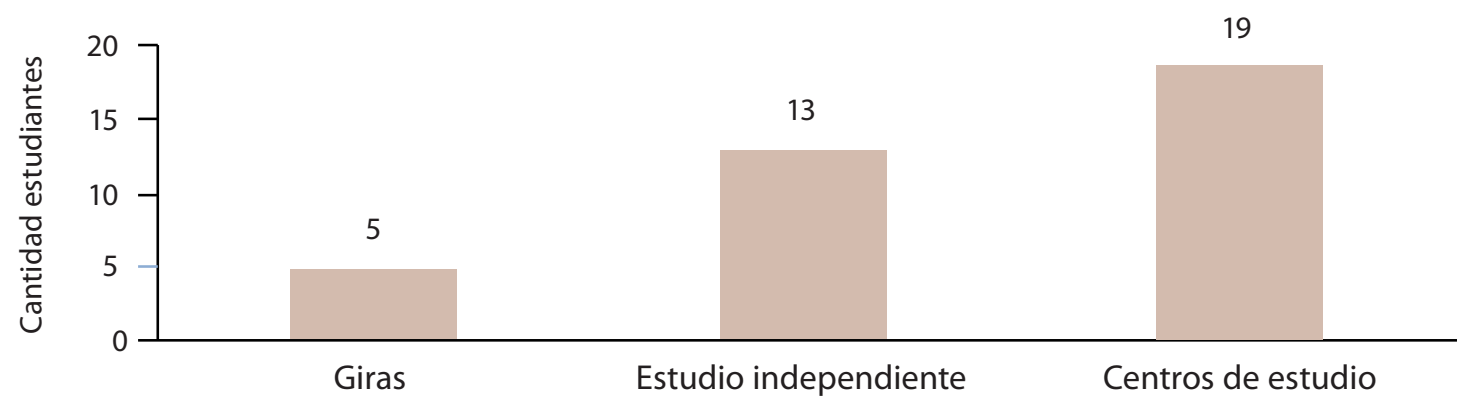

Tipos de actividades extracurriculares de preparación académica

Figura 4. Actividades extracurriculares de preparación académica realizadas preliminarmente a la participación en las OLICOCIBI por el estudiantado.

Nota: Datos recopilados del instrumento "Evaluación de los aportes cognitivos de la OLICOCIBI, dirigida a estudiantes" por los autores de este trabajo, julio, 2009.

Evidentemente, el desempeño sobresaliente y la consecuente mejoría cognitiva de temas biológicos se favorece, porque debido a su participación en las OLICOCIBI, el estudiantado se involucra y cumple con el desarrollo de diversas actividades de preparación que conllevan un reforzamiento del aprendizaje de los contenidos evaluados durante la competencia.

Ello significaría que el alumnado asociado a estas competencias dispone de más conocimientos científicos para: desenvolverse cotidianamente y resolver inconvenientes 0 necesidades, en comparación con otros estudiantes del Sistema Educativo Costarricense, lo que coincide con lo anunciado en la Conferencia Mundial sobre la Ciencia para el siglo XXI, según lo expresaron Gallopín, Funtowicz, O'Connor y Ravetz (2000), en referencia a imperativos de la AC.

En estudios posteriores, con la finalidad de, por ejemplo, construir recomendaciones para el gestionar del alumnado, podría detallarse el tiempo que dedican a la preparación, la constancia en su realización, el éxito o rendimiento ligado a su ejecución, etcétera.

Finalmente, toda la muestra afirmó que su participación en las OLICOCIBI refuerza y aporta conocimientos biológicos en su formación estudiantil. De igual manera, en las razones que justificaron su respuesta, se identificaron dos estrategias particulares que permiten tal contribución, desarrolladas durante el evento (figura 5). 


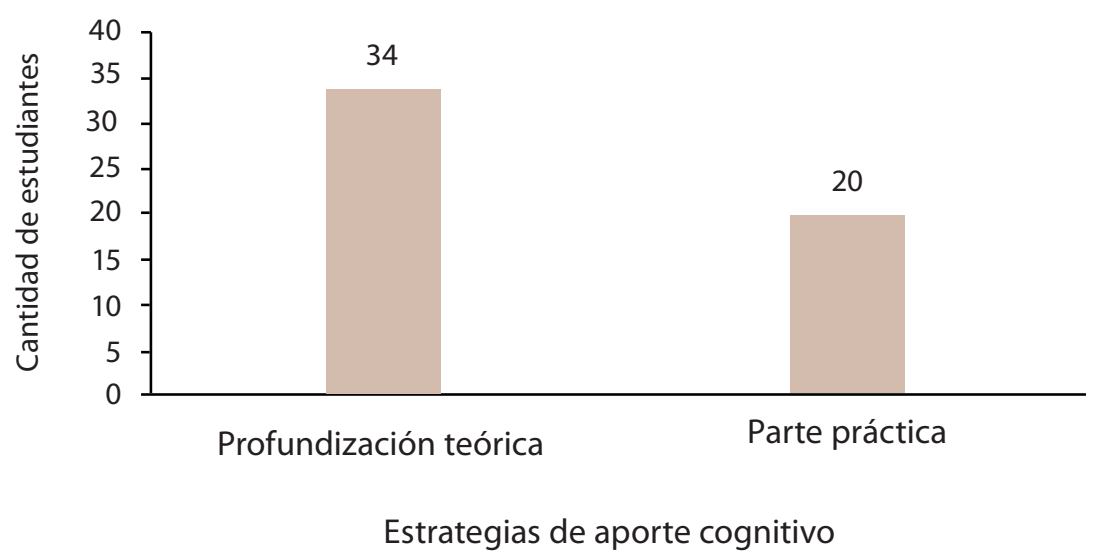

Figura 5. Estrategias que permiten reforzar y aportar conocimientos biológicos en el estudiantado mediante su participación en las OLICOCIBI.

Nota: Datos recopilados del instrumento "Evaluación de los aportes cognitivos de la OLICOCIBI, dirigida a estudiantes" por los autores de este trabajo, julio, 2009.

Así, la profundización en los contenidos teóricos del sistema de evaluación de las OLICOCIB y la temática, junto con la forma en la que se trabaja la parte práctica de esta competencia, se convierten en estrategias, directas o indirectas, que efectivamente permiten reforzar los aportes cognitivos en la AC del estudiantado y favorecen situaciones de enseñanza aprendizaje de la Biología (Vázquez et al., 2007).

Por lo tanto, el aumento en el rendimiento académico, el cumplimiento de variadas actividades para la preparación cognitiva, el aprendizaje de contenidos no abarcados por los temarios oficiales de estudio y la anuencia por incorporar nuevos temas a la evaluación teórica de las OLICOCIBI, son ejemplos claros de lo que se establece como prácticas para el apoyo de una dimensión conceptual de AC.

Especialmente porque estas servirán al alumnado para aplicar nociones científicas, diversificar su conocimiento teórico para resolver problemas, investigaciones y situaciones reales o simuladas -tales como las propuestas en los exámenes y sesiones de campo-, estudiar la naturaleza y práctica de la Ciencia, además de actualizar conocimientos de la Biología (hechos, conceptos y teorías), como lo explicaron diversos autores (Furió et al., 2001; Marco-Stiefel, 2000; Vázquez et al. 2007).

\section{Conclusiones}

La AC se ha tratado de implementar en el gesticular de las corrientes pedagógicas y didácticas relacionadas con las Ciencias y puede estudiarse desde diversas perspectivas. Particularmente, se trabajó con la dimensión conceptual o cognitiva de su accionar para analizar los aportes de la misma derivados de la participación de estudiantes de secundaria en una actividad de extensión universitaria que promueve competencias académicas cognitivas orientadas hacia la Biología: las OLICOCIBI. 
URL: http://www.una.ac.cr/educare

Las contribuciones cognitivas en la AC del estudiantado, reconocidas a través de esta investigación, fueron:

Se genera un mejor manejo cognitivo de las temáticas evaluadas durante la fase final, lo que evidencia un mejor rendimiento académico. Sumado a ello, se logra un compromiso por la actualización académica en las participantes y los participantes, apoyado por el desarrollo de diversas labores de preparación previa para participar en este concurso.

La incorporación de contenidos no contemplados por el MEP supone un estudio de los mismos y preparación académica previa, por lo que se presumió que, además, son un aporte inédito, directo y exclusivo en la población participante del Sistema Educativo de Secundaria, que fortalece su dimensión cognitiva en las Ciencias Biológicas.

Se identificó un aumento en la anuencia por optimizar y diversificar el manejo cognitivo en este evento, primordialmente, por la noción generada de que permite ampliar conocimientos y extender el nivel de calidad educativa en su contexto.

Finalmente, se incorporan estrategias y acciones que servirán de base, a las distintas entidades involucradas en la ejecución de las OLICOCIBI, para dar continuidad a los aportes al mejoramiento y a la construcción de la dimensión conceptual de la AC en el estudiantado participante durante todas las ediciones de esta competencia, como por ejemplo:

- Al Ministerio de Educación Pública:

- Brindar mayor apoyo a eventos científicos extracurriculares para facilitar la asistencia del estudiantado en las fechas de convocatoria respectiva, dentro de la calendarización anual educativa; de este modo se estaría generando, en sus participantes, una sensación de amparo en su participación en las diversas gestiones que surjan desde estas competencias.

- Incorporar en el currículo educativo, tanto de primaria como de secundaria, de manera transversal o directa, las dimensiones y sentidos de AC para generar en el estudiantado un aprendizaje realmente significativo de las Ciencias, particularmente la Biología.

- Al Ministerio de Ciencia y Tecnología:

- Continuar con el apoyo financiero a este tipo de eventos y dar continuidad y cobertura, económica y logística, a las capacitaciones docentes generadas en las OLICOCIBI, como estrategia de retroalimentación en el crecimiento de la dimensión conceptual de AC en el estudiantado.

- A la Universidad Nacional:

- Sustentar económica y académicamente las OLICOCIBI para fortalecer el proceso de AC generado a partir de este proyecto. 
- A la Escuela de Ciencias Biológicas:

- Colaborar en las actividades de AC gestadas desde las OLICOCIBI (como capacitaciones docentes, guía de prácticas experimentales de la fase final, elaboración de pruebas escritas y otras) que requieran de la experiencia, de la formación profesional e investigativa de sus académicos (as) para diversificar las propuestas y dar un nivel mayor de calidad.

- A la Comisión Organizadora de la OLICOCIBI:

- Crear más espacios anuales que sirvan para fortalecer la dimensión cognitiva de AC en el profesorado para que se genere una retroalimentación en el estudiantado y, por lo tanto, en el nivel de competencia en las OLICOCIBI.

- Incluir otras estrategias evaluativas, en las secciones escritas de la fase eliminatoria y la final, que permitan una mejor apreciación de las temáticas que requieren mayor apoyo para su resolución en el estudiando y, también, la identificación de avances cognitivos en el manejo de los tópicos evaluados.

- A las instituciones educativas de secundaria:

- Premiar y reconocer la labor de estudiantes participantes, debido a las actividades de preparación, y a los logros obtenidos. Esto constituye una herramienta de avance en la enseñanza y el aprendizaje de la Biología, la aplicación de técnicas científicas y la práctica de actitudes o valores promovidos en la AC, en cada aula y centro educativo.

- Al profesorado participante:

- Diversificar la ejecución de actividades de apoyo que fomenten el proceso de enseñanza aprendizaje de la Biología y que se evidencien en el estudiantado por su desempeño y motivación por participar en las OLICOCIBI, lo cual respondería a una herramienta para promover la construcción de la dimensión cognitiva de AC.

- Participar activamente de las actualizaciones profesionales en tópicos biológicos y de los espacios de discusión, primordialmente los brindados en las OLICOCIBI, para fortalecer la dimensión cognitiva y, consecuentemente, el diálogo alfabetizador con su alumnado.

- Al estudiantado participante:

- Participar activamente de competencias académicas extracurriculares, sin importar el ámbito de preparación profesional en el cual se desempeñarán a corto y mediano plazo, debido a que les proveen habilidades, conocimientos, destrezas y actitudes que les permiten un correcto ejercicio social en cualquier área.

- Desarrollar estrategias autodidactas y en coordinación con otros (as) compañeros (as) para el estudio y compresión de los temas que serían evaluados en las OLICOCIBI. De este modo, mejorar su rendimiento y aumentar la capacidad cognitiva biológica como recurso de $A C$. 
URL: http://www.una.ac.cr/educare

- A futuros (as) investigadores (as):

- Documentar características referentes a los procesos de preparación previos de estas competencias, para brindar sugerencias o estrategias que podrían aumentar el desempeño de sus participantes.

- Generar otras investigaciones sobre el desarrollo de la AC en Costa Rica, en distintos ámbitos (en otras competencias académicas, instancias gubernamentales, etcétera), que incluso pudiesen dar seguimiento a este trabajo.

\section{Referencias}

Acevedo, J. A., Vázquez, Á. y Manassero, M. A. (2003). Papel de la educación CTS en una alfabetización científica y tecnológica para todas las personas. Revista electrónica de enseñanza de las ciencias, 2 (2), 80-111. Recuperado de http://www.saum.uvigo.es/reec/ volumenes/volumen2/Numero2/Art1.pdf

Aguilar, T. (1999). Alfabetización científica y educación para la ciudadanía. Madrid: Narcea.

Alvira, F. (1983). Perspectiva cualitativa-perspectiva cuantitativa en la metodología sociológica. REIS, 22, 53-75.

Barrantes, R. (1999). Investigación un camino al conocimiento. Un enfoque cuantitativo y cualitativo. San José, Costa Rica: EUNED.

Bourdieu, P., Chamboredon, J.-C. y Passeron, J.-C. (2002). El oficio del sociólogo. Presupuestos epistemológicos (1a ed. francés, 1973; Trad. F. H. Azcurra) México: Siglo Veintiuno.

Bybee, R. (1997). Towards an Understanding of Scientific Literacy [Hacia el entendimiento de la alfabetización científica]. En W. Graeber, y Bolte, C. (Eds.), Scientific Literacy: An International Symposium [Alfabetización Científica: Simposio Internacional] (pp. 37-68). Kiel: IPN.

Cajas, F. (2001). Alfabetización científica y tecnológica: La transposición didáctica del conocimiento tecnológico. Enseñanza de las Ciencias, 19(2), 243-254.

Carrizo, G., Irureta-Goyena, P. y López, E. (2000). Manual de fuentes de información. Zaragoza, España: Cegal.

Collins, A. (1997). National Science Education Standards: Looking backward and Forward [Estándares de la educación científica nacional: Mirando hacia atrás y hacia delante]. Elementary School Journal, 97(4), 299-313.

Declaración de Budapest. (1999). Marco general de acción de la Declaración de Budapest. Recuperado de http://www.oei.org.co/cts/budapest.dec.htm 
Dirección nacional de gestión curricular y formación docente. Ministerio de Educación, Ciencia y Tecnología de la República Argentina (2007). Proyecto de Alfabetización Científica. Buenos Aires, Argentina: Autor. Recuperado de http://repositorio.educacion.gov.ar:8080/dspace/ bitstream/handle/123456789/96070/EL002095.pdf?sequence=1

Fensham, P. (1985). Science for all: A reflective essay [Ciencia para todos: Un ensayo reflectivo]. Journal of curriculum studies, 17(4), 415-435. doi: 10.1080/0022027850170407

Furió, C., Vilches, A., Guisasola, J. y Romo, V. (2001). Finalidades de la enseñanza de las Ciencias en la secundaria obligatoria. ¿Alfabetización científica o preparación propedéutica? Enseñanza de las Ciencias, 19(3), 365-376. Recuperado de http://ensciencias.uab.es/ revistes/19-3/365-376.pdf

García, C. (1987). Producción y transferencia de paradigmas teóricos en la investigación socioeducativa. Caracas: Fondo Editorial Tropykos.

Giroux, H. (1997). Cruzando límites. Trabajadores culturales y políticas educativas. Barcelona: Paidós Ibérica.

Hernández, R., Fernández, C. y Baptista, P. (2003). Metodología de la investigación. México: McGraw-Hill. México.

Hicks, D. y Holden, C. (1995). Exploring the Future: A Missing Dimension in Environmental Education [Explorando el futuro: Un faltante en la dimensión ambiental de educación]. Environmental Education Research, 1(2), 185-193.

Johnson, J. (1989). Technology: Report of the Project 2061 Phase I Technology Panel [Tecnología: Reporte del proyecto 2061 fase I panel de tecnología]. Washington, DC: American Association for the Advancement of Science.

Kemp, A. (2002). Implications of Diverse Meanings for 'Scientific Literacy' [Implicaciones de los diversos significados de alfabetización científica]. En P. A. Rubba, J. A. Rye, W. J. DiBiase y B. A. Crawford (Eds.), Proceedings of the 2002 Annual International Conference of the Association for the Education of Teachers in Science [Memoria de la Conferencia Anual de la Asociación de Educadores de Ciencias 2002] (pp. 1202-1229). Charlotte, North Carolina: Association for the Education of Teachers in Science.

Laugksch, R. (2000). Scientific Literacy: A Conceptual Overview [Alfabetización científica: Un resumen conceptual]. Science Education, 84(1), 71-94. doi: 10.1002/(SICI)1098237X(200001)84:1<71::AID-SCE6>3.0.CO;2-C

Marco-Stiefel, B. (2000). La alfabetización científica. En F. J. Perales y P. Cañal (Coords.), Didáctica de las ciencias experimentales. Teoría y práctica de la enseñanza de las Ciencias (141-164). Alcoi: Marfil. 
URL: http://www.una.ac.cr/educare

Martínez, M. (2001). Comportamiento humano. Nuevos métodos de investigación (2a ed). México: Editorial Trillas.

Miller, J. (Spring, 1983). Scientific Literacy: A Conceptual and Empirical Review [Alfabetización científica: Una revisión conceptual y empírica]. Daedalus, 112(2), 29-48. Recuperado de http://www.jstor.org/stable/i20024848

Olimpiadas Costarricenses de Ciencias Biológicas (OLICOCIBI). (2011). Normativa. Heredia, Costa Rica: Universidad Nacional. Recuperado de http://www.una.ac.cr/olicocibi/index. php?option=com remository\&ltemid=27\&func=startdown\&id=61

Reid, D. J. y Hodson, D. (1993). Ciencia para todos en secundaria. Madrid: Narcea. Recuperado de http://books.google.es/books?id=qjpXI03th7gC\&printsec=frontcover\&hl=es\&source= gbs ge summary $r \& c a d=0 \# v=$ onepage $\& q \& f=f a l s e$

Reyes, L. y Molina, A. (2005). Alfabetización científica: creencias, roles, metas y contextos para un mundo mejor. Enseñanza de las Ciencias. Número extraordinario, 1-4.

Shamos, M. (1995). The Myth of Scientific Literacy [El mito de la alfabetización científica]. New Brunswick, NJ: Rutgers University Press.

Vázquez, Á., Manassero, M. A., Acevedo, J. A. y Acevedo, P. (2007). Consensos sobre la naturaleza de la Ciencia: La comunidad tecnocientífica. Revista electrónica de enseñanza de las Ciencias, 6(2), 331-363. 